\title{
The Intensification of the Process
}

of Opening Thrust Middlings Refining Industry

(Part-4. Optimization of the Interaction

\author{
of Persistent Industrial Products Refinery Production \\ of Barium Per-Oxide After \\ their Joint Mechanical Activation)
}

\author{
Evgeniy A. Pavlov*a, \\ Edward V. Maltseva, Andrey A. Guschinskiy ${ }^{a}$, \\ Aleksandr P. Skuratov ${ }^{b}$ and Sergey V. Belyaev ${ }^{b}$ \\ ${ }^{a}$ «Krastsvetmet», JSC \\ 1 Transportny proezd, Krasnoyarsk, Russia \\ ${ }^{b}$ Siberian Federal University \\ 79 Svobodny, Krasnoyarsk, Russia
}

Received 20.01.2016, received in revised form 09.02.2016, accepted 22.04.2016

The paper formulated the basic principle of technological refining of platinum metals, their transfer into the solution at the first stage. Ways of transfer of platinum metals in soluble form, a method of intensification of pro-process. Justified and selected factors has a key influence on the process of the preliminary mechanical activation and subsequent sintering of hard middlingbl refining barium peroxide. Proposed and applied a flexible method of planning the study, which combines an element of uncertainty with a certain deterministic description of the statistical results. Six-factor mathematical model of the optimized process are obtained, the correlation coefficient of which with the experiment is 0.92. The optimal parameters and performed their experimental testing.

Keywords: refining of platinum metals, mechanical activation, sintering, experimental design, mathematical model, optimization.

Citation: Pavlov E.A., Maltsev E.V., Guschinskiy A.A., Skuratov A.P., Belyaev S.V. The intensification of the process of opening thrust middlings refining industry (Part-4. Optimization of the interaction of persistent industrial products refinery production of barium per-oxide after their joint mechanical activation), J. Sib. Fed. Univ. Eng. technol., 2016, 9(5), 758-769. DOI: 10.17516/1999-494X-2016-9-5-758-769.

(C) Siberian Federal University. All rights reserved

* Corresponding author E-mail address: EPavlov@knfmp.net 


\title{
Интенсификация процесса
}

вскрытия упорных промпродуктов

аффинажного производства.

(Ч. 4. Оптимизация процесса взаимодействия

упорных промпродуктов аффинажного производства

с перекисью бария после их совместной механоактивации)

\author{
Е.А. Павлов ${ }^{\text {a }, ~ Э . В . ~ М а л ь ц е в ~}{ }^{\text {, }}$ \\ А.А. Гущинский ${ }^{\mathrm{a}}$, А.П. Скуратов \\ ${ }^{a} \mathrm{OAO}$ «Красиветмет» \\ Россия, 660027, Красноярск, Транспортный проезд, 1 \\ ${ }^{6}$ Сибирский федеральный университет \\ Россия, 660041, Красноярск, Свободный, 79
}

\begin{abstract}
В статьесформулированосновнойтехнологическийпринцип аффинажаплатиновыхметаллов, перевод их в раствор на первом этапе. Обсуждаются пути перевода платиновых металлов в растворимую форму, предложен способ интенсификации процесса. Обоснованы и выбраны факторы, оказывающие ключевое влияние на прочесс предварительной механоактивации и последующего спекания упорного промпродукта аффинажа с перекисью бария. Предложен и применен гибкий метод планирования исследования, в котором сочетается элемент неопределенности с определенной долей детерминированного описания статистических результатов. Получена шестифакторная математическая модель оптимизируемого прочесса, коэффициент корреляции которой с экспериментом составляет 0,92. Определень оптимальные параметры и проведена их экспериментальная апробация.
\end{abstract}

Ключевые слова: аффинаж платиновых металлов, механоактиваџия, спекание, планирование эксперимента, математическая модель, оптимизация.

В современной технологии аффинажа платиновых металлов основным принципом, лежащим в её основе, является предварительный перевод их в раствор. Если для Pt и Pd данный процесс с физико-химической и технологической точек зрения не представляет особых трудностей, то для металлов-спутников платины (далее - MCП) Rh, Ir, Ru этот процесс вызывает определенные сложности, связанные с низкой эффективностью и дороговизной применяемых способов [1].

Известен аналитический прием перевода металлов-спутников платины $\mathrm{Rh}, \mathrm{Ir}, \mathrm{Ru}$ в pacтворимые формы путем предварительного спекания смеси их с реагентом-окислителем перекисью бария [2]. Степень прямого извлечения суммы целевых компонентов МСП в раствор при разумном как с экономической, так и технологической точек зрения соотношении масс промпродукта и перекиси бария $2,5 \div 3,0$ составляет $75 \div 85$ масс. \%, что является одним из наиболее высоких показателей, достигаемых за один цикл. Данный способ один из наиболее эффективных и широко применяется в промышленном масштабе. Однако он из-за распада Советского государства и нарушения ранее установленных связей, остановки отдельных химических производств был заменен на другие, менее эффективные. 
Способ спекания упорных промпродуктов аффинажного производства с перекисью бария, несмотря на высокий расход реагента и недостаточную полноту извлечения в раствор МСП, в настоящее время широко применяется в аналитической практике, а задача, решение которой направлено на интенсификацию данного процесса с целью снижения расхода дорогого реагента и повышения полноты перевода целевых компонентов в растворимые формы, по-прежнему является актуальной.

Выполненные ранее исследования [3] показали, что предварительная механоактивация упорных промпродуктов аффинажного производства не приводит к значительному повышению полноты перевода платиновых металлов в растворимые формы при спекании с перекисью бария. Также авторами установлено, что механоактивация оказывает существенное влияние на физико-химические свойства перекиси бария, сопровождается повышением её химической активности и увеличением гомогенизации концентрата с химическим реагентом [4]. Поэтому дальнейшая работа была направлена на исследование совместной механоактивационной обработки упорного промпродукта, содержащего МСП с перекисью бария, и влияния различных факторов, характеризующих процессы механоактивации и спекания, на полноту и скорость перевода платиновых металлов в растворимые формы.

В качестве упорного материала для исследования был взят (две пробы) один из промпродуктов аффинажного производства - тяжелый сплав спутников. Содержание платиновых металлов в пробах представлено в табл. 1, а характеристика реагента - перекиси бария - приведена в работе [4].

На основании анализа физико-химических основ процесса механоактивации [3], а также результатов по исследованию влияния механоактивации на структурные и физико-химические свойства перекиси бария [4] следует, что значительный вклад в избыточную энергию обрабатываемого материала вносят дефекты кристаллической структуры. Механизм накопления дефектов в твердом теле во время механоактивации реализуется в том числе и через пластическую деформацию, сопровождаемую образованием дислокаций, движение которых, особенно когда дислокации находятся в разных плоскостях, приводит к образованию точечных дефектов и искажений в структуре. При этом доля пластической деформации в общей деформации частиц обрабатываемого материала зависит от предельной нагрузки, связанной с ускорением нагружения, величина которого определяется технологическим параметром механоактивации ускорением мелющих тел (далее - УМТ) в единицах $\mathrm{g}=9,8$ м $/ \mathrm{c}^{2}$ [5]. Используемое в настоящих исследованиях оборудование, планетарная мельница АИР - 0,015М и АГО - 2, позволяло достичь величины УМТ в пределах от 1 до 45 g (табл. 2).

Таблица 1. Содержание платиновых металлов, используемых для проведения исследований, в упорных промпродуктах аффинажного производства

\begin{tabular}{|c|c|c|c|c|c|c|}
\hline \multirow{2}{*}{$\begin{array}{c}\text { Наименование } \\
\text { порного промпродукта }\end{array}$} & \multicolumn{5}{|c|}{ Содержание платиновых металлов, \% масс. } \\
\cline { 2 - 8 } & $\mathrm{Pt}$ & $\mathrm{Pd}$ & $\mathrm{Rh}$ & $\mathrm{Ir}$ & $\mathrm{Ru}$ & $\Sigma \mathrm{MПГ}$ \\
\hline Тяжелый сплав спутников №1 & 3,3 & 4,9 & 10,8 & 3,2 & 7,6 & 29,8 \\
\hline Тяжелый сплав спутников №2 & 3,2 & 7,4 & 16,3 & 4,7 & 8,4 & 40,0 \\
\hline
\end{tabular}

гМПГ* - сумма металлов платиновой группы. 
Таблица 2. Значения варьируемых факторов при проведении исследований

\begin{tabular}{|c|c|c|c|c|c|}
\hline \multirow{2}{*}{ Факторы } & \multicolumn{5}{|c|}{ Уровни } \\
\hline & 1 & 2 & 3 & 4 & 5 \\
\hline X & 1 & 2 & 3 & 4 & 5 \\
\hline $\begin{array}{l}\mathrm{X}_{2} \text {-отношение массы обрабатываемого } \\
\text { материала к массе мелющих тел; отн.ед. }\end{array}$ & 0,05 & 0,05 & 0,1 & 0,2 & 1,0 \\
\hline $\mathrm{X}_{3}$-ускорение мелющих тел; g=9,8 м/с² & 1 & 25 & 35 & 45 & 45 \\
\hline $\begin{array}{l}\mathrm{X}_{4} \text {-отношение массы упорного промпродукта к } \\
\text { массе перекиси бария; отн.ед. }\end{array}$ & 0,67 & 0,5 & 0,5 & 0,4 & 0,4 \\
\hline X5-температура спекания; ${ }^{\circ} \mathrm{C}$ & 900 & 900 & 800 & 700 & 500 \\
\hline X6-продолжительность спекания; ч & 0,5 & 1,0 & 1.5 & 2,0 & 2,0 \\
\hline
\end{tabular}

Частота следования импульсов механического воздействия на частицы обрабатываемого материала в рабочей камере механоактиватора подчиняется законам статистического распределения. В работе [6] была сделана попытка определить статистическую модель измельчения в энергонапряженных мельницах и ввести для всех частиц обрабатываемого материала величину средней частоты следования импульсов механического воздействия. Показано, что с уменьшением величины отношения массы обрабатываемого материала к массе мелющих тел средняя частота следования импульсов механического воздействия возрастает. В результате установлено, что кроме указанной выше величины фактором, определяющим эффективность влияния механоактивации на физико-химические свойства обрабатываемого материала, является отношение массы обрабатываемого материала к массе мелющих тел, значение которого в наших исследованиях изменяли от 1 до 0,05 (табл. 2).

Увеличение продолжительности механоактивации пропорционально росту количества импульсов механического воздействия и степени гомогенизации обрабатываемых компонентов. В связи с этим в качестве одного из факторов, характеризующих процесс механоактивации, была выбрана продолжительность обработки. Продолжительность механоактивации варьировали от 0 до 5 мин (табл. 2). Выбор верхней границы продолжительности обработки - 5 мин. - обусловлен тем, что за это время механоактивации один из обрабатываемых компонентов - перекись бария - запасает до 80 \% избыточной энергии от возможного её количества при продолжительности механоактивации 30 мин [4].

Изменение свойств поверхностного слоя частиц МСП [7], структурных и физикохимических свойств перекиси бария [4], а также высокая степень гомогенизации смеси при механоактивационной обработке позволяют сделать предположение о возможном снижении значений параметров процесса спекания: температуры, продолжительности и расхода реагента - перекиси бария. Границами для изменения расхода реагента были выбраны: верхняя $1: 2,5$ (минимальное количество, применяемое на практике), нижняя 1 : 1,5 (предполагаемое, минимально необходимое количество реагента).

В табл. 2 приведены выбранные и обоснованные для оптимизации процесса взаимодействия упорных промпродуктов аффинажного производства с перекисью бария после их механоактивации факторы, а также диапазон их изменения. 
Температуру спекания ограничили значениями: нижнее $\left(500^{\circ} \mathrm{C}\right)$ - начало разложения перекиси бария, верхнее $\left(900^{\circ} \mathrm{C}\right)$ - рекомендуемое максимальное значение для устойчивой работы промышленной камерной печи КО-9.

Продолжительность спекания варьировали от 0,5 до 2,0 ч при промежуточных значениях (табл. 2), что гораздо меньше продолжительности спекания по ранее действующей в промышленности технологии (10 ч) и близко к рекомендуемой в аналитике.

Выбранные рабочие интервалы основных технологических параметров позволяют получать отличающиеся в широком диапазоне значения степени извлечения платиновых металлов.

Изучение сложных систем (в нашем случае - последовательное протекание двух независимых процессов: механоактивация и спекание) требует учитывать множество различных факторов (табл. 2). При этом, в случае детерминированного моделирования, большое количество учитываемых факторов приводит к значительному затягиванию процесса их согласования. Применение же вероятностного подхода ограничивается «загрублением» математической модели, и остается возможность выхода расчетного значения за физические или логические пределы изучаемого объекта (превышение 100 \%-ного извлечения или получения отрицательных величин для выхода продукта). Поэтому нами применен более гибкий метод планирования исследования, в котором сочетается существенный элемент неопределенности с долей детерминированного описания статистических результатов [8].

На основании выбранного вероятностно-детерминированного метода с учетом предложенных шести факторов составлен план шестифакторного эксперимента на пяти уровнях (табл. 3).

Таблица 3. Матрица шестифакторного эксперимента

\begin{tabular}{|c|c|c|c|c|c|c|c|}
\hline $\begin{array}{c}\text { Номер } \\
\text { опыта }\end{array}$ & $\mathrm{X}_{1}$ & $\mathrm{X}_{2}$ & $\mathrm{X}_{3}$ & $\mathrm{X}_{4}$ & $\mathrm{X}_{5}$ & $\mathrm{X}_{6}$ & $\mathrm{y}_{9}$ \\
\hline 1 & 2 & 3 & 4 & 5 & 6 & 7 & 8 \\
\hline 1 & 1 & 0,05 & 1 & 0,67 & 900 & 0,5 & 60,33 \\
\hline 2 & 1 & 0,1 & 35 & 0,5 & 800 & 1,5 & 79,41 \\
\hline 3 & 1 & 0,05 & 25 & 0,5 & 900 & 1,0 & 85,79 \\
\hline 4 & 1 & 1 & 45 & 0,4 & 500 & 2,0 & 60,03 \\
\hline 5 & 1 & 0,2 & 45 & 0,4 & 700 & 2,0 & 75,87 \\
\hline 6 & 3 & 0,05 & 35 & 0,5 & 500 & 2,0 & 59,83 \\
\hline 7 & 3 & 0,1 & 25 & 0,4 & 700 & 0,5 & 70,71 \\
\hline 8 & 3 & 0,05 & 45 & 0,4 & 900 & 1,5 & 92,80 \\
\hline 9 & 3 & 1 & 45 & 0,67 & 800 & 1,0 & 59,74 \\
\hline 10 & 3 & 0,2 & 1 & 0,5 & 900 & 2,0 & 84,14 \\
\hline 11 & 2 & 0,05 & 25 & 0,4 & 800 & 2,0 & 89,12 \\
\hline 12 & 2 & 0,1 & 45 & 0,67 & 900 & 2,0 & 74,22 \\
\hline 13 & 2 & 0,05 & 45 & 0,5 & 500 & 0,5 & 39,46 \\
\hline
\end{tabular}


Продолжение табл. 3

\begin{tabular}{|c|c|c|c|c|c|c|c|}
\hline 1 & 2 & 3 & 4 & 5 & 6 & 7 & 8 \\
\hline 14 & 2 & 1 & 1 & 0,5 & 700 & 1,5 & 65,09 \\
\hline 15 & 2 & 0,2 & 35 & 0,4 & 900 & 1,0 & 92,09 \\
\hline 16 & 5 & 0,05 & 45 & 0,5 & 700 & 1,0 & 64,98 \\
\hline 17 & 5 & 0,1 & 45 & 0,5 & 900 & 2,0 & 85,85 \\
\hline 18 & 5 & 0,05 & 1 & 0,4 & 800 & 2,0 & 72,56 \\
\hline 19 & 5 & 1 & 35 & 0,4 & 900 & 0,5 & 77,84 \\
\hline 20 & 5 & 0,02 & 25 & 0,67 & 600 & 1,5 & 30,38 \\
\hline 21 & 4 & 0,05 & 45 & 0,4 & 900 & 1,5 & 93,84 \\
\hline 22 & 4 & 0,1 & 1 & 0,4 & 500 & 1,0 & 48,72 \\
\hline 23 & 4 & 0,05 & 35 & 0,67 & 700 & 2,0 & 66,21 \\
\hline 24 & 4 & 1 & 25 & 0,5 & 900 & 2,0 & 82,32 \\
\hline 25 & 4 & 0,2 & 45 & 0,5 & 800 & 0,5 & 68,68 \\
\hline
\end{tabular}

Каждая строка матрицы экспериментов (табл. 3) определяет собой конкретные условия их проведения. По этим условиям были проведены опыты и сделаны соответствующие извлечения платиновых металлов в раствор $\left(y_{\ni_{i}}\right)$ из упорного промпродукта. Содержание платиновых металлов приведено в табл. 1 под номером 1. Выборка из этих результатов на точечные зависимости для каждого фактора $\left(Y_{i}-f\left(X_{i}\right)\right.$, где $y_{i}$ - экспериментальные значения степени извлечения суммы металлов платиновой группы, $X_{i}$ - определенный фактор), осуществляется следующим образом: для определенного фактора на всех уровнях выбираются результаты, соответствующие его конкретному единственному значению, последние складываются и делятся на число слагаемых.

При таком подходе происходит усреднение действия всех остальных факторов, так как каждый из них принимает в этих опытах все пять своих уровней и таким образом сам себя компенсирует нижними (слабыми) и верхними (сильными) значениями. Этот принцип соблюдается для каждого уровня любого фактора. В результате получается система (1) из шести функций, являющихся частными зависимостями от выбранных факторов:

$$
\left\{\begin{array}{l}
y_{1}=f\left(X_{1}\right)=73,44-0,81\left(X_{1}-3\right)^{2} \\
y_{1}=f\left(X_{1}\right)=73,44-0,81\left(X_{1}-3\right)^{2} \\
y_{2}=f\left(X_{2}\right)=72,49-3,59\left(X_{2}-3\right)^{1 / 2} \\
y_{3}=f\left(X_{3}\right)=66,17+1,18\left(X_{3}-3\right)^{1 / 2} \\
y_{4}=f\left(X_{4}\right)=77,36-71,30\left(X_{4}-0,4\right) \\
y_{5}=f\left(X_{5}\right)=0,093 X_{5} \\
y_{6}=f\left(X_{6}\right)=X_{6} /\left(8,012 \cdot 10^{-4}+0,013 X_{6}\right)
\end{array}\right.
$$

Расчет коэффициента корреляции $R$ и его значимости $T_{R}$ для найденных частных функций вели по формулам: 


$$
\begin{aligned}
& R=\sqrt{1-\frac{(n-1) \prod_{i=1}^{n}\left(y_{J_{i}}-Y_{T_{i}}\right)^{2}}{(n-k-1) \sum_{i=1}^{n}\left(y_{\Im_{i}}-Y_{T_{i}}\right)^{2}},} \\
& T_{R}=\frac{R \sqrt{n-k-1}}{1-R^{2}},
\end{aligned}
$$

где $n$ - число описываемых точек; $k$ - число действующих факторов; $y_{\ni_{i}}$ - экспериментальное значение результата; $y_{T_{i}}$ - расчетное значение [8].

Результаты расчета $R$ и $T_{R}$ приведены в табл. 4 .

Значимость коэффициента корреляции и проверяемой зависимости для $95 \%$-ного уровня достоверности определяется условием [9] $T_{R}>2$, из которого следует, что все они значимы (табл. 4).

В рассматриваемом методе матричного планирования для обработки статистических данных использовали формулу (4), представленную в работе [10]:

$$
y_{\Pi}=\frac{\prod_{i=1}^{k} y_{i}}{y_{c p}^{k-1}},
$$

где $Y_{\text {П }}$ - многофакторная функция Протодьяконова [10]; $y_{i}-$ частная функция; $k$ - число факторов (частных функций); $V_{c p}$ - среднее значение всех учитываемых результатов эксперимента.

С учетом значений всех частных функций уравнение (4) будет иметь следующий вид:

$$
\begin{aligned}
& y_{I I}=\frac{1}{71,5^{5}}\left[73,44-0,81\left(X_{1}-3\right)^{2}\right]\left[72,49-3,59 \sqrt{X_{2}-0,05}\right]\left[66,17+1,18 \sqrt{X_{3}-1}\right] \times \\
& \times\left[77,36-71,30\left(X_{4}-0,4\right)\right]\left[0,093 X_{5}\right]\left[\frac{X_{6}}{8,012 \cdot 10^{-4}+0,013 X_{6}}\right],
\end{aligned}
$$

Сопоставлением экспериментальных значений ( $y_{\ni}$ - табл. 3$)$, соответствующих конкретным условиям эксперимента, и расчетных $\left(V_{\Pi}\right)$ по уравнению (5), полученных после подстановки в уравнение значений факторов, также соответствующих конкретным условиям эксперимента, получили соотношение между ними (рис. 1).

Таблица 4. Коэффициент корреляции и его значимость для частных функций

\begin{tabular}{|c|c|c|c|}
\hline Частная функция & $R$ & $T_{R}$ & Значимость функции \\
\hline $\mathrm{y}_{1}$ & 0,70 & 2,75 & + \\
\hline $\mathrm{y}_{2}$ & 0,92 & 8,27 & + \\
\hline $\mathrm{y}_{3}$ & 0,79 & 2,92 & + \\
\hline $\mathrm{y}_{4}$ & 0,99 & 49,75 & + \\
\hline $\mathrm{y}_{5}$ & 0,99 & 86,17 & + \\
\hline $\mathrm{y}_{6}$ & 0,99 & 61,42 & + \\
\hline
\end{tabular}




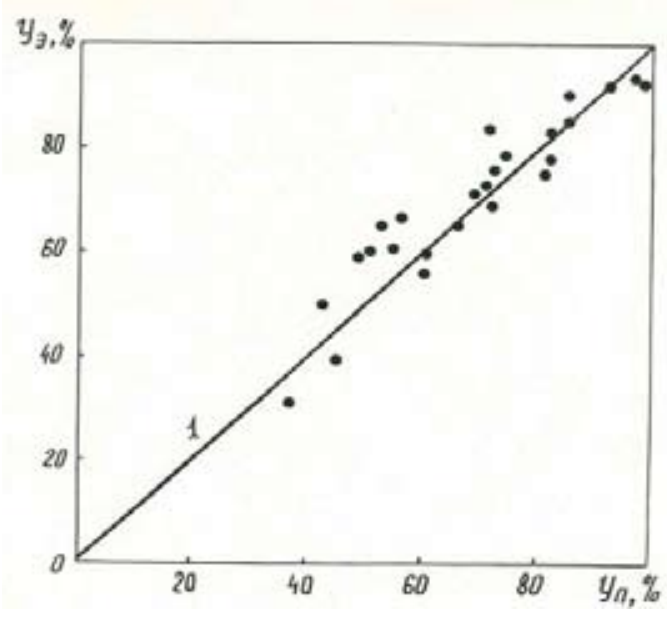

Рис. 1. Соотношение экспериментальных и расчетных по уравнению (5) данных для извлечения суммы платиновых металлов в раствор; $1-Y_{\ni}=Y_{\text {П }}-$ идеальная зависимость

Сравнение точек соотношения с идеальной зависимостью $y_{\ni}=y_{\text {II }}$ показывает их равномерный разброс над и под ней, что свидетельствует о высокой степени согласованности расчетных значений, полученных на основе математической модели (5), с экспериментальными данными. Коэффициент корреляции многофакторной зависимости (5) с экспериментальными данными, рассчитанный по уравнению (2), составил $R=0,92$, что подтверждает хорошее согласование расчетных и экспериментальных данных.

Далее, на основе полученной математической модели проанализированы и определены оптимальные параметры для процесса взаимодействия упорных промпродуктов аффинажного производства с перекисью бария после их совместной механоактивации.

Анализируя влияние факторов, характеризующих процесс механоактивации: продолжительность механоактивации, отношение массы обрабатываемого материала к массе мелющих тел, ускорение мелющих тел (энергонапряженность), на полноту спекания упорных промпродуктов с перекисью бария, видим, что все они по сравнению со спеканием без механоактивации приводят к более полному переводу платиновых металлов в растворимую форму. На рис. 2 показано влияние факторов (параметров) механоактивации на полноту извлечения МПГ в растворимую форму.

В связи с тем, что уравнение (5) является шестифакторным, выполнен разрез по четырем переменным, закрепленным в точках экстремума их частных функций для системы уравнений (1).

Для продолжительности механоактивации (рис. $2 a$ ) максимальный вклад в повышение степени извлечения отмечается при обработке шихты в течение 3 мин. При этом увеличение фактора энергонапряженности до $15 \div 20$ g приводит к заметному повышению степени извлечения. Однако дальнейшее его увеличение на степень извлечения влияет слабо.

Наиболее значимо среди факторов механоактивации (табл. 4) отношение массы обрабатываемого материала к массе мелющих тел. По результатам анализа (рис. 2б) он также оказывает существенное влияние на повышение степени извлечения МПГ в растворимую форму. 


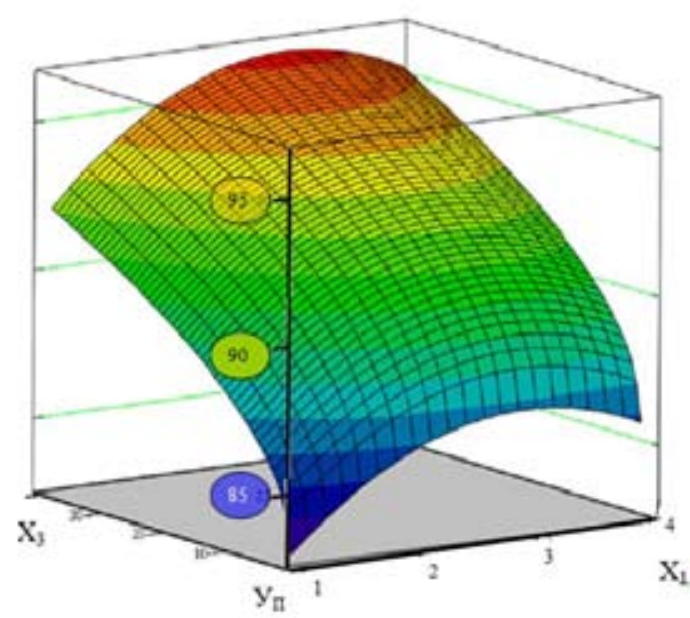

a

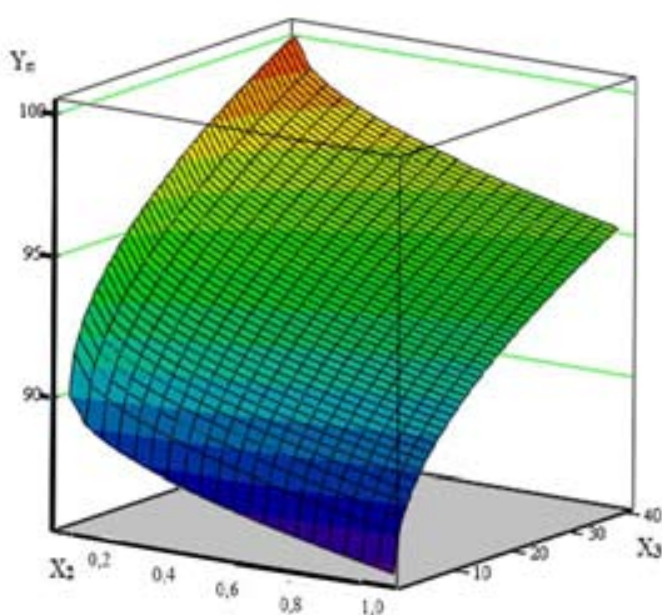

6

Рис. 2. Зависимость растворения спека МПГ с перекисью бария: а - от продолжительности механоактивации $\left(X_{1}\right)$, ускорение мелющих тел $\left(X_{3}\right)$. Разрез выполнен в точках: $X_{2}=0,05 ; X_{4}=0,4$; $X_{5}=900 ; X_{6}=2 ; 6$ - от отношения массы обрабатываемого материала к массе мелющих тел $\left(X_{2}\right)$, ускорение мелющих тел $\left(X_{3}\right)$. Разрез выполнен в точках: $X_{1}=3 ; X_{2}=0,05 ; X_{5}=900 ; X_{6}=2$

Установленная зависимость на исследуемом параметрическом диапазоне носит возрастающий характер. Ограничивающим фактором ее непрерывного повышения является экономическая целесообразность, так как в пределе мы достигаем отношения бесконечно малой массы смеси упорного промпродукта с перекисью бария к массе мелющих тел.

Анализ влияния факторов, характеризующих отношение масс упорного промпродукта и перекиси бария, процесс спекания упорного промпродукта с перекисью бария после их совместной механоактивации (продолжительность спекания, температура спекания), проводился по зависимостям (рис. 3), построенным на основании шестифакторной математической модели (5), целевой функцией в которой служит полнота перевода МПГ в растворимую форму. Разрез выполнен по четырем переменным, закрепленным в точках экстремума их частных функций для системы уравнений (1).

Установленное влияние температуры и продолжительности спекания на степень извлечения платиновых металлов (рис. $3 a$ ) не противоречит общепринятым представлениям об их роли в твердофазных процессах, поэтому с увеличением каждого из них наблюдается рост целевой функции.

Учитывая, что масса упорного промпродукта во всех опытах постоянна, зависимость степени извлечения платиновых металлов от отношения массы упорного промпродукта к массе перекиси бария (рис. 3б) отражает влияние расхода перекиси бария на полноту спекания с ней. Повышение расхода реагента от 1/1,5 до 1/2,5 приводит к росту степени извлечения платиновых металлов, максимальная величина которой без механоактивации составляет около 80 \%. После механоактивации та же степень извлечения достигается при меньшем расходе реагента $(1 / 1,7 \div 1 / 1,5)$, а при расходе $1 / 2,5$ наблюдается практически полный перевод платиновых металлов в растворимые формы. 


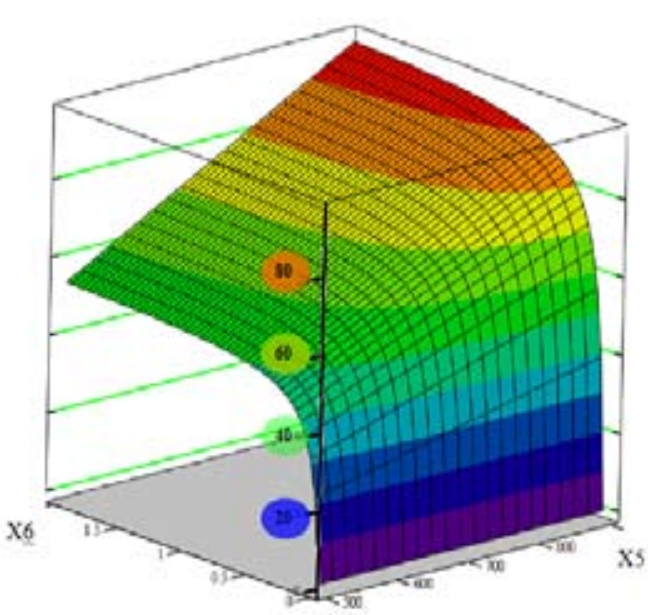

$\mathrm{Yn}$

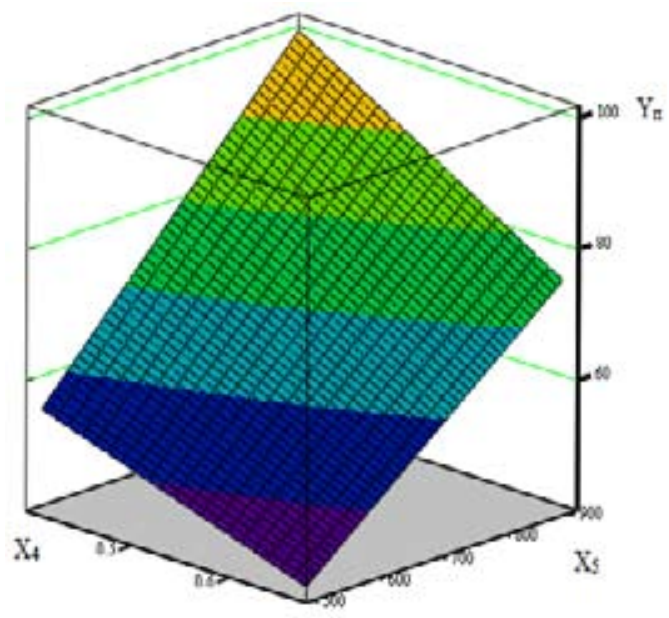

б

Рис. 3. Зависимость растворения спека МПГ с перекисью бария: а - от температуры спекания $\left(X_{5}\right)$, продолжительность спекания $\left(X_{6}\right)$. Разрез выполнен в точках: $X_{1}=3 ; X_{2}=0,05 ; X_{3}=40 ; X_{4}=0,4 ;$ б - от отношения массы упорного промпродукта к массе перекиси бария $\left(X_{4}\right)$, температуры спекания $\left(X_{5}\right)$. Разрез выполнен в точках: $X_{1}=3 ; X_{2}=0,05 ; X_{3}=40 ; X_{6}=2$

Таблица 5. Оптимальные значения параметров процесса взаимодействия упорных промпродуктов аффинажного производства с перекисью бария после их совместной механоактивации

\begin{tabular}{|c|c|}
\hline Факторы & Значение \\
\hline $\mathrm{X}_{1}$ - продолжительность механоактивации; мин & 3 \\
\hline $\mathrm{X}_{2}$ - отношение массы обрабатываемого материала к массе мелющих тел; отн.ед. & 0,05 \\
\hline $\mathrm{X}_{3}$ - ускорение мелющих тел (энергонапряженность); g=9,8 м/с² & 45 \\
\hline $\mathrm{X}_{4}$ - отношение массы упорного промпродукта к массе перекиси бария; отн.ед. & 0,4 \\
\hline $\mathrm{X}_{5}$ - температура спекания; ${ }^{\circ} \mathrm{C}$ & 900 \\
\hline $\mathrm{X}_{6}-$ продолжительность спекания; ч & 2 \\
\hline
\end{tabular}

На основании выполненного анализа поведения и взаимного влияния определенных нами факторов, характеризующих в установленных интервалах процессы механоактивации и спекания упорных промпродуктов аффинажного производства с перекисью бария, были определены для них оптимальные значения, при которых целевая функция - степень перевода МПГ в растворимые формы - достигает своей максимальной величины (табл. 5).

\section{Выводы}

Таким образом, в оптимальных условиях для экспериментального подтверждения установленных оптимальных параметров была проведена серия из пяти опытов на упорном промпродукте, содержание платиновых металлов в котором приведено в табл. 1, ТСС №2. Показано, что извлечение МПГ в раствор составляет 97,6 99,0 \% (среднее 98,5 \%). Проверка результатов

$$
-767-
$$


исследований проводилась в присутствии сотрудников института «Сибцветметниипроект», лаборатория ФХМА.

\section{Список литературы}

[1] Павлов Е.А., Мальцев Э.В., Гущинский А.А. Энергосберегающий способ перевода в растворимую форму металлов-спутников платины. Известия Самарского научного иентра PAH, 2012, 14 (4(5), 1273-1276 [Pavlov E.A., Maltsev E.V., Gushchinskii A.A. Energy-saving way to convert a soluble form of the metals platinum satellites. Proceedings of the Samara Scientific Center of the Russian Academy of Sciences, 2012, 14 (4(5), 1273-1276 (in Russian)]

[2] Бимиш Ф.Е. Аналитическая химия благородных металлов: в 2 ч. М.: Мир, 1969, 698 с. [Bimish F.E. Analytical chemistry of noble metals. Moscow, Mir, 1969, 698 p. (in Russian)]

[3] Павлов Е. А. Совершенствование технологии вскрытия упорных промпродуктов аффинажного производства с применением механоактивации. Автореф. дис. ... канд. техн. наук. Красноярск, 1990, 28 c. [Pavlov E.A. Improving the technology of tamper resistant industrial products refining production with the use of mechanical activation. Krasnoyarsk, 1990, 28 p. (in Russian)]

[4] Павлов Е.А., Мальцев Э.В., Гущинский А.А. и др. Интенсификация процесса вскрытия упорных промпродуктов аффинажного производства: Ч. 2. Влияние механоактивации на структуру и реакционную способность перекиси бария. Известия Самарского научного иентра РАН, 2013, 15 (6(2), 438-442 [Pavlov E.A., Maltsev E.V., Gushchinskii A.A. et al. The intensification of the process of opening resistant industrial products refining production. Part 2. The influence of mechanical activation on the structure and reactivity of peroxide of barium. Proceedings of the Samara Scientific Center of the Russian Academy of Sciences, 2013, 15 (6(2), 438-442 (in Russian)]

[5] Авакумов Е.Г. Механические методы активачии химических прочессов. Новосибирск: Наука, 1979. 254 с. [Avakumov E.G. Mechanical methods of activation of chemical processes. Novosibirsk, Nauka, 1979. 254 p. (in Russian)]

[6] Восель С.В., Васенин Н.Т., Помощников Э.В. и др.. Статистическая модель измельчения и активации в аппаратах с мелющими телами. Препринт ИК СО РАН. Новосибирск, 1985, 32 c. [Vosel' S.V., Vasenin N.T., Pomoshchnikov E.V. et al. A statistical model of the grinding and activation in the apparatus with grinding bodies. Novosibirsk, 1985, 32 p. (in Russian)]

[7] Павлов Е.А., Мальцев Э.В., Гущинский А.А. Интенсификация процесса вскрытия упорных промпродуктов аффинажного производства: Ч.1. Влияние механоактивации на структуру и реакционную способность металлов спутников платины и материалов их содержащих. Известия Самарского научного центра РАН, 2013, 15 (6(6), 432-437 [Pavlov E.A., Maltsev E.V., Gushchinskii A.A. et al. The intensification of the process of opening resistant industrial products refining production. Part 1 . The influence of mechanical activation on the structure and reactivity of the metals platinum satellites, and materials containing them. Proceedings of the Samara Scientific Center of the Russian Academy of Sciences, 2013, 15 (6(6), $432-437$ (in Russian)]

[8] Малышев В.Н. Вероятностно-детерменированное планирование эксперимента. АлмаАта: Наука, 1981. 116 с. [Malyshev V.N. Probability-determenirovana experiment planning. AlmaAta, Nauka, 1981. 116 p. (in Russian)] 
[9] Сиськов В.И. Корреляиионный анализ в экономических исследованиях. М.: Наука, 1975, 162 c. [Siskov V.I. Correlation analysis in economic research. Moscow, Nauka, 1975, 162 p. (in Russian)]

[10] Протодьяконов М.М., Тедер Р.И. Методика раџионального планирования эксперименma. М.: Наука, 1970, 76 c. [Protod'iakov M.M., Teder R.I. The methodology of rational planning of the experiment. Moscow, Nauka, 1970, 76 p. (in Russian)] 